\title{
Activity of c-Met/ALK Inhibitor Crizotinib and Multi-Kinase VEGF Inhibitor Pazopanib in Metastatic Gastrointestinal Neuroectodermal Tumor Harboring EWSR1-CREB1 Fusion
}

\author{
Vivek Subbiah $^{\mathrm{a}} \quad$ Oliver Holmes $^{\mathrm{b}} \quad$ Kyle Gowen $^{\mathrm{b}}$ Daniel Spritz ${ }^{\mathrm{b}}$ Behrang Amini ${ }^{\mathrm{a}}$ \\ Wei-Lien Wang $^{a} \quad$ Alexa B. Schrock $^{b}$ Funda Meric-Bernstam ${ }^{a}$ Ralph Zinner $^{a}$ \\ Sarina Piha-Paul ${ }^{a}$ Maria Zarzour ${ }^{a}$ Julia A. Elvin ${ }^{b}$ Rachel L. Erlich ${ }^{b}$ \\ David L. Stockman ${ }^{c}$ Jo-Anne Vergilio ${ }^{b}$ James H. Suh ${ }^{b}$ Philip J. Stephens ${ }^{b}$ \\ Vincent Miller $^{b}$ Jeffrey S. Ross ${ }^{b, d}$ Siraj M. Ali ${ }^{b}$ \\ ${ }^{a}$ The University of Texas MD Anderson Cancer Center, Houston, Tex., ${ }^{b}$ Foundation Medicine, Inc., Cambridge, Mass., \\ 'OrthoPath Labs, Orthopaedic and Sarcoma Associates PC, Kalamazoo, Mich., and d Albany Medical College, \\ Albany, N.Y., USA
}

\section{Key Words}

Case report - Crizotinib · Pazopanib - Gastrointestinal neuroectodermal tumor - Clear-cell sarcoma - EWSR1 . CREB1 · Comprehensive genomic profiling · Genomic profiling

\begin{abstract}
Malignant gastrointestinal neuroectodermal tumor (GNET) is an aggressive rare tumor, primarily occurring in young adults with frequent local-regional metastases and recurrence after local control. The tumor is characterized by the presence of EWSR1-ATF1 or EWSR1-CREB1 and immunohistochemical positivity for $\mathrm{S}-100$ protein without melanocytic marker positivity. Due to poor responses to standard sarcoma regimens, GNET has a poor prognosis, and development of effective systemic therapy is desperately needed to treat these patients. Herein, we present a patient with a small
\end{abstract}

\section{KARGER}

E-Mail karger@karger.com www.karger.com/ocl bowel GNET who experienced recurrent hepatic and skeletal metastases after a primary resection. Comprehensive genomic profiling (CGP) in the course of clinical care with DNA and RNA sequencing demonstrated the presence of an exon 7 to exon 6 EWSR1-CREB1 fusion in the context of a diploid genome with no other genomic alterations. In a clinical trial, the patient received a combination of $250 \mathrm{mg}$ crizotinib with $600 \mathrm{mg}$ pazopanib quaque die and achieved partial response and durable clinical benefit for over 2.8 years, and with minimal toxicity from therapy. Using a CGP database of over 50,000 samples, we identified 11 additional cases that harbor EWSR1-CREB1 and report clinicopathologic characteristics, as these patients may also benefit from such a regimen.

(c) 2016 S. Karger AG, Basel (c) 2016 S. Karger AG, Basel

0030-2414/16/0916-0348\$39.50/0
Dr. Vivek Subbiah

Department of Investigational Cancer Therapeutics, Unit 455

The University of Texas MD Anderson Cancer Center

1515 Holcombe Blvd, Houston, TX 77030 (USA)

E-Mail vsubbiah@mdanderson.org 


\section{Background}

Malignant gastrointestinal neuroectodermal tumor (GNET), also known as clear-cell sarcoma (CCS)-like tumor of the gastrointestinal tract (CCSLGT), is a highly aggressive rare malignant tumor originating from the gastrointestinal tract that has a median survival of 18.5 months $[1,2]$. Patients commonly present with regional lymph node and hepatic metastases and frequently recur [1-3]. GNET has features that occasionally overlap histologically with CCS of tendons and aponeuroses, including the immunohistochemical expression of S-100 protein [4]. However, CCS of tendons and aponeuroses often shows positivity for melanocytic markers (HMB45, melan-A, and tyrosinase), whereas GNET is often negative for melanocytic markers and is positive for synaptophysin [5]. These tumors share common genetic alterations: chromosomal translocation events $\mathrm{t}(12 ; 22)$ (q13; $\mathrm{q} 12)$ and $\mathrm{t}(2 ; 22)(\mathrm{q} 34 ; \mathrm{q} 12)$, leading to chimeric fusion proteins EWSR1-ATF1 and EWSR1-CREB1, respectively $[3,6]$.

Standard treatment regimens for CCS and CCSLGT include resection with regional lymph node dissection, radiotherapy, and chemotherapeutic regimens that include ifosfamide and doxorubicin but carry a poor prognosis. Herein, we present a case of metastatic GNET harboring EWSR1-CREB1 with an unexpected partial response and durable clinical benefit to treatment with combined crizotinib and pazopanib exceeding 2 years.

\section{Methods}

The original liver biopsy sample and post-relapse biopsy samples were submitted as FFPE blocks for comprehensive genomic profiling (CGP) [7]. Using FoundationOne Heme, DNA of 405 cancer-related genes plus select introns from 31 genes involved in rearrangements was sequenced to a median depth of coverage of $481 \times ; 265$ of these genes also went through RNA sequencing.

\section{Results}

\section{Case Presentation}

A 27-year-old female initially presented with symptoms of abdominal pain, thought to be due to cholecystitis. During cholecystectomy in October 2010, a liver tumor was observed, and intraoperative biopsies were performed. Pathology noted small round cell tumor morphology. She underwent an exploratory laparotomy with a right hepatectomy, placement of gold fiducials in the left lobe of the liver, small bowel resection, abdominal lymphadenectomy, and placement of an On-Q pain pump. Pathology from the surgical specimen revealed multifocal metastatic sarcoma to the liver. Her small bowel specimen was diagnosed as CCS of the bowel with transmural extension. She had micrometastatic sarcoma (less than $1 \mathrm{~mm}$ ) in $1 / 21$ mesenteric lymph nodes (see fig. 1 for pathology reviewed at MD Anderson).

Final pathology revealed a diagnosis of GNET with metastatic disease in both the liver and 1/21 mesenteric lymph nodes. Two months later, the left liver was noted to be free of disease after a follow-up MRI. A year later, additional whole body imaging showed the presence of a left hepatic lobe hypermetabolic lesion, which was biopsied 6 months later; pathology showed this to be a hematoma. A year later, a PET scan revealed metastatic lesions in the lung, liver, and ilium. Subsequent palliative radiotherapy with 30 Gy in 10 fractions was completed in November 2013.

A rearrangement between chromosomes 22 and 2 was found giving rise to $E W S R 1-C R E B 1$ comprised of EWSR1 exons 1-7 and CREB1 exons 6-8 (see fig. 2). In addition, three variants of unknown significance were identified; CTCF P315S, NSD1 V2584L and RARA I258fs*13. The tumoral genome was otherwise assessed as diploid, and no other genomic alterations were identified in the sample.

The patient was enrolled in a trial of daily $250 \mathrm{mg}$ crizotinib plus $600 \mathrm{mg}$ pazopanib (clinicaltrials.gov No. NCT01548144). After the first cycle of treatment, the patient showed an early response, and therapy continued to display clinical benefit of partial response per RECIST for 1.5 years (see fig. 3). At that time, a lump that was thought to be progressive hematoma was noted and was completely excised to reveal CCS. CGP was performed on the recurrent CCSLGT and harbored the same EWSR1-CREB1 fusion with low-level amplification $(3 \times)$ of several genes, but no additional known driver or resistance mutations were identified. The patient continues on therapy for a total of 2.8 years from start of therapy.

In reviewing 45,632 carcinomas, 3,803 sarcomas, and 1,526 melanomas assayed with CGP in the course of clinical care, we identified 11 additional cases that harbor EWSR1-CREB1 fusions. These patients were 5 males and 6 females with a mean age of 34 (range 13-51). The tumors carried the diagnoses as follows: 5 sarcomas not otherwise specified (2 of which originated from the small intestine), 2 GNETs, 2 malignant neoplasms of 

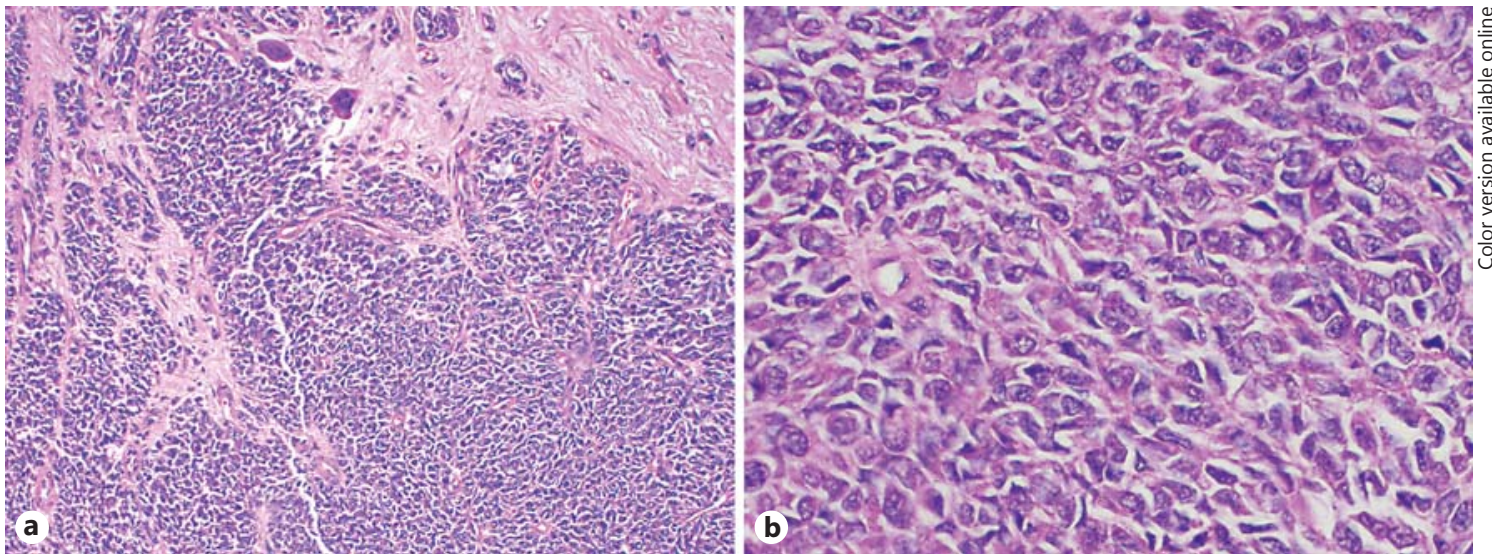

Fig. 1. a Cellular tumor composed of epithelioid cells, rare giant cells, arranged in variably sized nests and separated by fibrous septae (H\&E, 10× magnification). b High power reveals the tumor is composed of epithelioid cells with oval nuclei and vesicular chromatin (H\&E, 40× magnification). Tumor was positive for S-100 protein while being negative for pan-melanocytic cocktail.

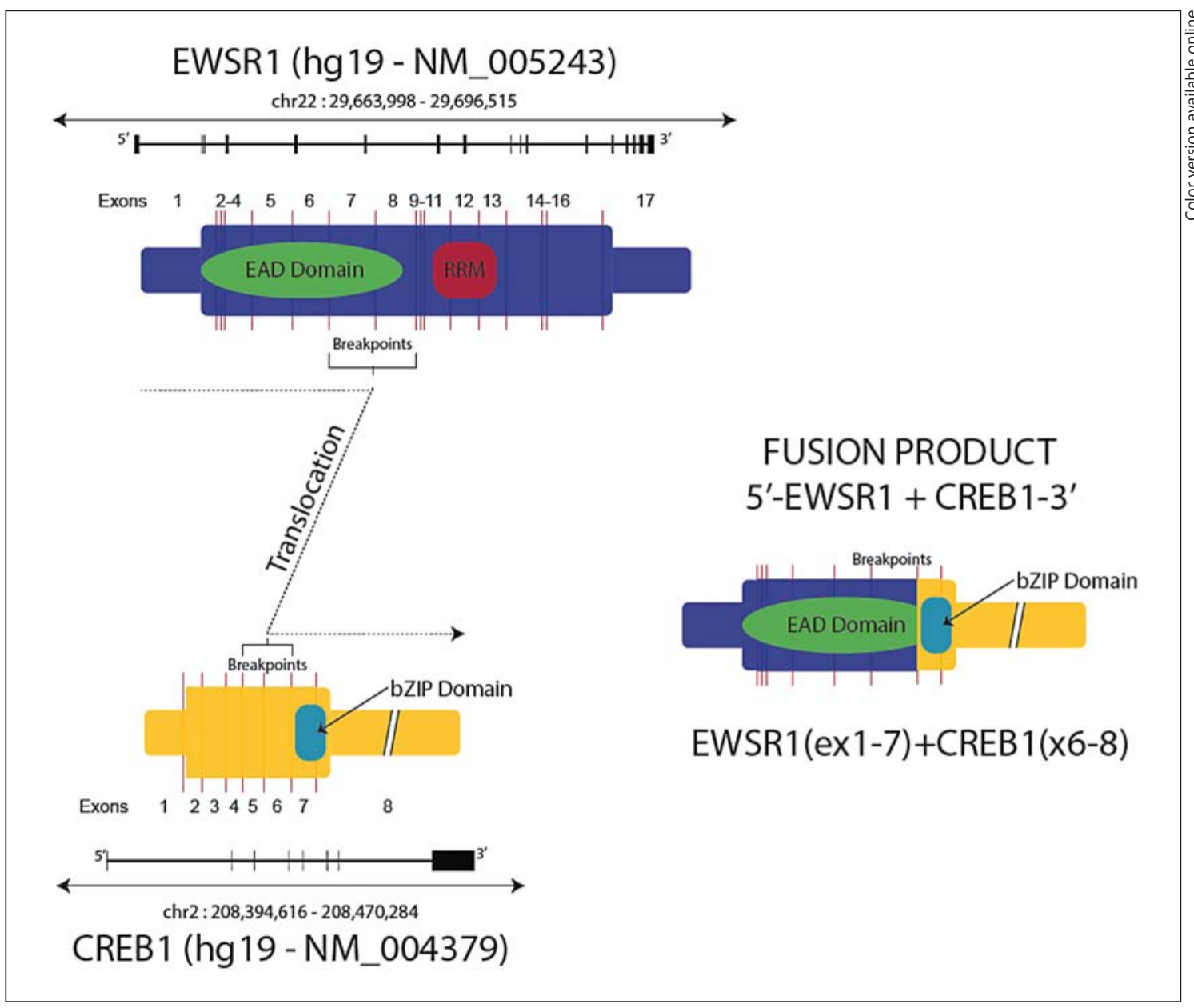

Fig. 2. Cartoon showing the EWSR1, CREB1 and the EWSR1-CREB1 fusion product. 


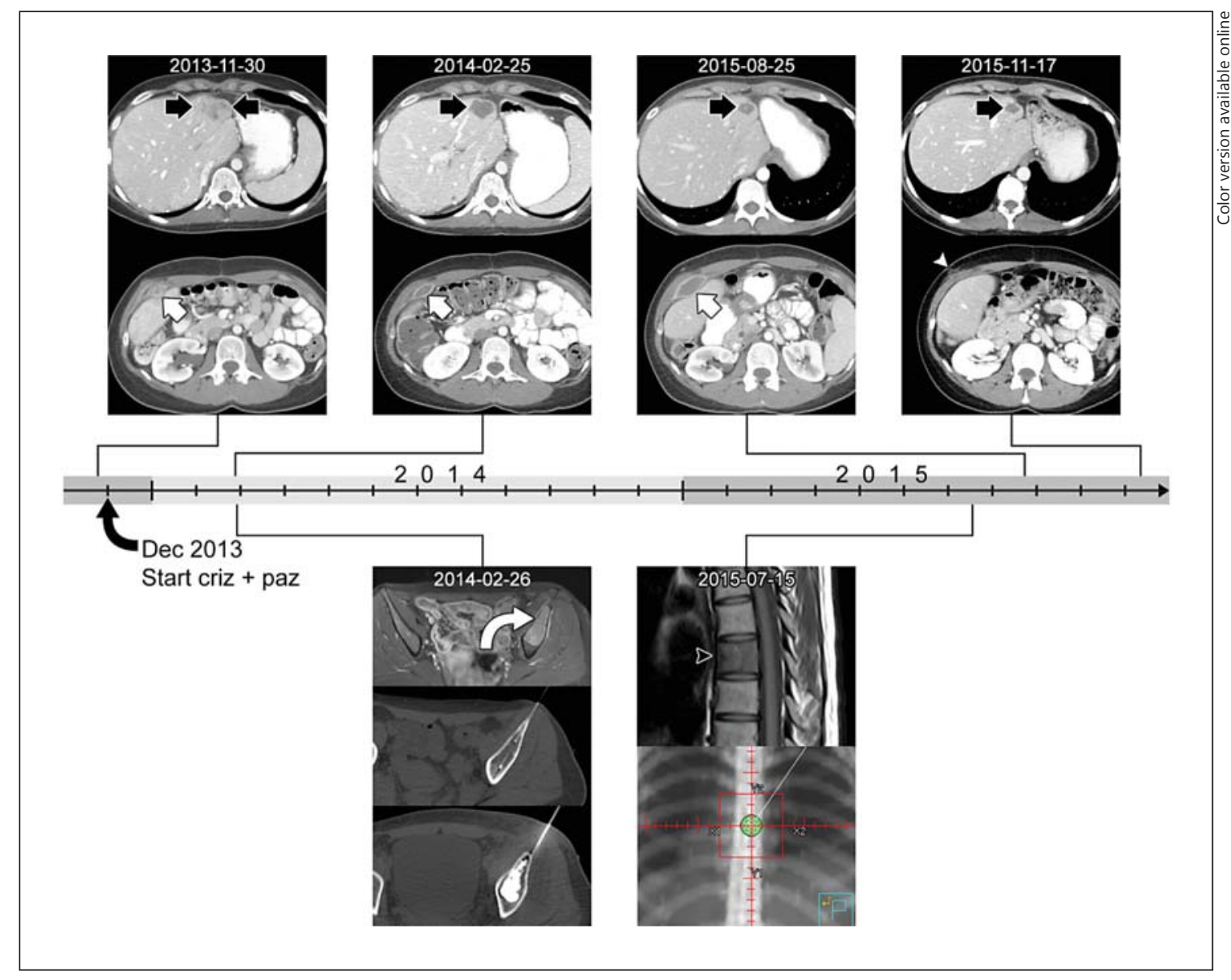

Fig. 3. Time course of imaging response following initiation of crizotinib and pazopanib in early December 2013. The CT images above the timeline show the response of a liver lesion (black arrow) and an abdominal wall lesion (white arrow) to therapy. The liver lesion had heterogeneous enhancement at the baseline CT from November 2013. On follow-up imaging, it decreased in size and degree of enhancement, developing central low attenuation representing necrosis. The abdominal wall lesion also had heterogeneous enhancement at baseline and decreased in degree of enhancement on subsequent CTs. In contrast to the liver lesion, it enlarged and became symptomatic, and was finally resected in

unknown primary, 1 melanoma, and 1 head and neck mucoepidermoid carcinoma. All but one case harbored fusions between EWSR1 exons 1-7/8 and CREB1 exons 6/7-8. The melanoma case fused EWSR1 exons 1-7 with CREB1 exons 5-8. With the exception of one sarcoma, which harbored $C D K N 2 A / B$ homozygous deletion, there was a very low prevalence of other known mutations, none of which were in drivers of oncogenesis, implicating EWSR1-CREB1 fusion as the main driver in these cases.

GNET with Pazopanib and Crizotinib
September 2015. Postoperative CT from November 2015 shows the surgical scar (white arrowhead). The images below the timeline show other lesions that underwent local control. In February 2014 contrast-enhanced T1-weighted MR image shows a bone metastasis in the left ilium (curved arrow, top row). This subsequently underwent cryoablation (middle row) followed by cement augmentation (bottom row) to reduce risk of pathological fracture. In July 2015, a metastasis was detected on MRI at T7 (black arrowhead on T1-weighted image). This lesion underwent palliative radiation to a dose of 2,000 cGy in 5 fractions (bottom row, radiation therapy planning image).

\section{Conclusions}

Herein, we present the first case of a GNET patient harboring EWSR1-CREB1 who exhibited a durable nearcomplete response to crizotinib and pazopanib for 1.5 years. The durable response to pazopanib combined with crizotinib presented in this case study was unanticipated. The current paradigm of advanced cancer benefitting from targeted therapy relies on the presence of genomic alterations that predict response, such as in the case of 
non-small cell lung cancer that harbors oncogenic drivers such as ALK fusions and EGFR mutations [8,9]. Inherent proof of the specificity of the pairing of therapy and genomic alteration is manifested in very specific mechanisms of acquired resistance, such as base substitutions in $A L K$ fusions and the emergence of T790M or MET amplification in EGFR mutant lung cancer [10-13].

This case may exist outside that paradigm, as EWSR1 fusions are currently not strongly predicted to be sensitizing to any targeted therapies. However, one group of studies reported durable, partial responses to sunitinib in extra-skeletal myxoid chondrosarcoma patients harboring the characteristic EWSR1-NRF4A3 but not TAF1-NRFA3 [14]. The initial observation was of the partial response of two patients, and then repeated in 8 more patients, with 6 having partial response, and 2 having stable disease. These paradigm-setting observations suggest that promiscuous multikinase inhibitors can be an effective treatment for a tumor driven by a specific EWSR1 fusion, although the mechanism of action remains unclear.

The case reported here raises questions of mechanism of action, applicability of this finding to other GNETs, and more broadly applicability to other tumors harboring EWSR 1 fusions. These questions can only be answered by further investigation, but some implications do arise from this single patient narrative.

How does the combination of pazopanib with crizotinib, a known MET inhibitor, produce a response in this GNET patient? EWSR1-ATF and EWSR1-CREB1 are both thought to upregulate the MET pathway via MITF and, thus, may confer a vulnerability to a MET inhibitor [15]. A recent report of interim results of a phase 2 EORTC study of crizotinib monotherapy in patients with METdriven (likely assessed by IHC) CCS demonstrated stable disease in 7/12 cases lasting approximately 5 months [16]. Although the predetermined response rate endpoint was not met in this trial, for the case at hand, the addition of pazopanib, a promiscuous multikinase inhibitor, to crizotinib, which may already confer some clinical benefit, could further inhibit downstream signaling. An additive, possibly synergistic effect, could explain the greater response here, both in terms of the magnitude of the response and the duration of 1.5 years. A similar effect was observed in a recent case study that reported a prolonged response ( $>6$ months) to the same therapeutic combination of crizotinib and pazopanib in a patient with an inflammatory myofibroblastic tumor harboring a DCTN1$A L K$ fusion, which was in contrast to other ALK+ inflammatory myofibroblastic tumors that have often not responded as well to crizotinib monotherapy [17]. It is unclear what the low-level amplification across multiple genes means as a mechanism of acquired resistance, but this relatively focal change is in keeping with resistance mechanisms observed with targeted therapy.

We additionally report other patients that harbor the same EWSR1-CREB1 fusion, with the thought that such patients may also benefit from the combination of crizotinib and pazopanib. This fusion was found in cases carrying a range of diagnoses, including 5 sarcomas (not otherwise specified), which inherently pose a diagnostic challenge as highlighted in a recent study [18]. The cases diagnosed as melanoma, with positivity for melanocytic markers, and mucoepidermoid carcinoma, with a head and neck anatomic location, could be reconceived as CCS and an EWSR1 rearranged tumor of the salivary gland, such as clear-cell carcinoma [19]. Regardless of diagnosis, such patients are likely eventually refractory to standard therapy and thus could benefit from an empiric challenge with the regimen described here.

More broadly, over 10 distinct EWSR1 fusions have been reported across a large range of tumor types, predominantly in sarcomas. Could these cases also respond to the combination regimen listed here? This question warrants investigation, as does assessment of monotherapy with tyrosine kinase inhibitors, such as sunitinib. Given that the length of time likely needed to definitively understand a possible mechanism of action is unclear, empiric investigation of treatment may be warranted, as these patients typically progress through standard of care regimens. However, it may be just as likely that there are fusion-specific therapeutic sensitivities, both depending on the partner to EWSR1.

We have reported a case of GNET harboring an EWSR1-CREB1 fusion in a patient who showed a partial response to combination therapy with the receptor tyrosine kinase inhibitors crizotinib and pazopanib, with clinical benefit for over 2.8 years. To our knowledge, this represents the first report of a significant clinical response to combination targeted therapy in a tumor harboring an EWSR1-CREB1 fusion, and suggests possible benefit for other oncology patients harboring the same fusion, and potentially EWSR1 fusions generally.

\section{Acknowledgements}

The authors thank Pfizer ${ }^{\circledR}$ for providing crizotinib for the clinical trial. Supported in part by the Cancer Prevention Research Institute of Texas Grant (RP110584) and The University of Texas MD Anderson Cancer Center Support Grant (P30 CA016672). NCATS grant UL1 TR000371 (Center for Clinical and Translational Sciences). 


\section{Statement of Ethics}

Treatment and consent on the investigational trial and data collection were performed in accordance with the guidelines of The University of Texas MD Anderson Cancer Center Institutional Review Board. Clinical evaluation and assessments were performed per protocol. Written informed consent was obtained from the patient for enrolling on the clinical trial and for publication of this case report and any accompanying images. A copy of the written consent is available for review by the Editor-in-Chief of this journal.

\section{Disclosure Statement}

O.H., K.G., D.S., J.A.E., R.L.E., J.-A.V., J.H.S., P.J.S., V.M., J.S.R. and S.M.A. are employees of and have equity interest in Foundation Medicine, Inc.

\section{References}

1 Zambrano E, Reyes-Mugica M, Franchi A, Rosai J: An osteoclast-rich tumor of the gastrointestinal tract with features resembling clear cell sarcoma of soft parts: reports of 6 cases of a GIST simulator. Int J Surg Pathol 2003;11:75-81.

2 Stockman DL, Miettinen M, Suster S, Spagnolo D, Dominguez-Malagon $\mathrm{H}$, Hornick JL, Adsay V, Chou PM, Amanuel B, Vantuinen P, Zambrano EV: Malignant gastrointestinal neuroectodermal tumor: clinicopathologic, immunohistochemical, ultrastructural, and molecular analysis of 16 cases with a reappraisal of clear cell sarcoma-like tumors of the gastrointestinal tract. Am J Surg Pathol 2012; $36: 857-868$

- 3 Antonescu CR, Suurmeijer AJH, Zhang L, Sung Y-S, Jungbluth AA, Travis WD, Al-Ahmadie H, Fletcher CDM, Alaggio R: Molecular characterization of inflammatory myofibroblastic tumors with frequent ALK and ROS1 gene fusions and rare novel RET rearrangement. Am J Surg Pathol 2015;39:957967.

4 Enzinger FM: Clear-cell sarcoma of tendons and aponeuroses. An analysis of 21 cases. Cancer 1965; 18:1163-1174.

5 Chung EB, Enzinger FM: Malignant melanoma of soft parts. A reassessment of clear cell sarcoma. Am J Surg Pathol 1983;7:405-413.

-6 Gineikiene E, Seinin D, Brasiuniene B, Brazaitis A, Griskevicius L, Jakubauskas A: Clear cell sarcoma expressing a novel chimerical transcript EWSR1 exon 7/ATF1 exon 6. Virchows Arch Int J Pathol 2012;461:339_ 343.

-7 Frampton GM, Fichtenholtz A, Otto GA Wang K, Downing SR, He J, Schnall-Levin M, White J, Sanford EM, An P, Sun J, Juhn F, Brennan K, Iwanik K, Maillet A, Buell J, White E, Zhao M, Balasubramanian S, Terzic S, Richards T, Banning V, Garcia L, Mahoney $\mathrm{K}$, Zwirko Z, Donahue A, Beltran H, Mosquera JM, Rubin MA, Dogan S, Hedvat CV, Berger MF, Pusztai L, Lechner M, Boshoff C, Jarosz M, Vietz C, Parker A, Miller VA, Ross JS, Curran J, Cronin MT, Stephens PJ, Lipson D, Yelensky R: Development and validation of a clinical cancer genomic profiling test based on massively parallel DNA sequencing. Nat Biotechnol 2013;31:1023-1031.

8 Shaw AT, Kim D-W, Mehra R, Tan DSW, Felip E, Chow LQM, Camidge DR, Vansteenkiste J, Sharma S, De Pas T, Riely GJ, Solomon BJ, Wolf J, Thomas M, Schuler M, Liu G, Santoro A, Lau YY, Goldwasser M, Boral AL, Engelman JA: Ceritinib in ALK-rearranged nonsmall-cell lung cancer. N Engl J Med 2014; 370:1189-1197.

-9 Paez JG, Jänne PA, Lee JC, Tracy S, Greulich H, Gabriel S, Herman P, Kaye FJ, Lindeman N, Boggon TJ, Naoki K, Sasaki H, Fujii Y, Eck MJ, Sellers WR, Johnson BE, Meyerson M: EGFR mutations in lung cancer: correlation with clinical response to gefitinib therapy. Science 2004;304:1497-1500.

10 Ou S-H, Milliken JC, Azada MC, Miller VA, Ali SM, Klempner SJ: ALK F1174V mutation confers sensitivity while ALK I1171 mutation confers resistance to alectinib. The importance of serial biopsy post progression. Lung Cancer 2016;91:70-72.

-11 Ou S-HI, Greenbowe J, Khan ZU, Azada MC, Ross JS, Stevens PJ, Ali SM, Miller VA, Gitlitz B: I1171 missense mutation (particularly I1171N) is a common resistance mutation in ALK-positive NSCLC patients who have progressive disease while on alectinib and is sensitive to ceritinib. Lung Cancer 2015;88:231-234.

12 Balak MN, Gong Y, Riely GJ, Somwar R, Li AR, Zakowski MF, Chiang A, Yang G, Ouerfelli O, Kris MG, Ladanyi M, Miller VA, Pao W: Novel D761Y and common secondary T790M mutations in epidermal growth factor receptor-mutant lung adenocarcinomas with acquired resistance to kinase inhibitors. Clin Cancer Res Off J Am Assoc Cancer Res 2006; 12:6494-6501.

13 Bean J, Brennan C, Shih J-Y, Riely G, Viale A, Wang L, Chitale D, Motoi N, Szoke J, Broderick S, Balak M, Chang W-C, Yu C-J, Gazdar A, Pass H, Rusch V, Gerald W, Huang S-F, Yang P-C, Miller V, Ladanyi M, Yang C-H, Pao W: MET amplification occurs with or without T790M mutations in EGFR mutant lung tumors with acquired resistance to gefitinib or erlotinib. Proc Natl Acad Sci USA 2007;104:20932-20937.
-14 Stacchiotti S, Pantaleo MA, Astolfi A, Dagrada GP, Negri T, Dei Tos AP, Indio V, Morosi C, Gronchi A, Colombo C, Conca E, Toffolatti L, Tazzari M, Crippa F, Maestro R, Pilotti S, Casali PG: Activity of sunitinib in extraskeletal myxoid chondrosarcoma. Eur J Cancer 2014;50:1657-1664.

15 Davis IJ, McFadden AW, Zhang Y, Coxon A, Burgess TL, Wagner AJ, Fisher DE: Identification of the receptor tyrosine kinase c-Met and its ligand, hepatocyte growth factor, as therapeutic targets in clear cell sarcoma. Cancer Res 2010;70:639-645.

16 Schoffski P, Wozniak A, Stacchiotti S, et al: Activity of crizotinib (C) in patients (pts) with clear cell sarcoma (CCSA) in EORTC phase II trial 90101 'CREATE'. J Clin Oncol 2015; 33(suppl):10542. http://meetinglibrary.asco. org/content/144465-156 (accessed January 23, 2016)

-17 Subbiah V, McMahon C, Patel S, Zinner R, Silva EG, Elvin JA, Subbiah IM, Ohaji C, Ganeshan DM, Anand D, Levenback CF, Berry J, Brennan T, Chmielecki J, Chalmers ZR, Mayfield J, Miller VA, Stephens PJ, Ross JS, Ali SM: STUMP un'stumped': anti-tumor response to anaplastic lymphoma kinase (ALK) inhibitor based targeted therapy in uterine inflammatory myofibroblastic tumor with myxoid features harboring DCTN1-ALK fusion. J Hematol OncolJ Hematol Oncol 2015; 8:66.

18 Harris MH, DuBois SG, Glade Bender JL, Kim A, Crompton BD, Parker E, Dumont IP, Hong AL, Guo D, Church A, Stegmaier K, Roberts CWM, Shusterman S, London WB, MacConaill LE, Lindeman NI, Diller L, Rodriguez-Galindo C, Janeway KA: Multicenter feasibility study of tumor molecular profiling to inform therapeutic decisions in advanced pediatric solid tumors: the individualized cancer therapy (iCat) study. JAMA Oncol 2016, DOI: 10.1001/jamaoncol.2015.5689.

$\checkmark 19$ Skálová A, Weinreb I, Hyrcza M, et al: Clear cell myoepithelial carcinoma of salivary glands showing EWSR1 rearrangement: molecular analysis of 94 salivary gland carcinomas with prominent clear cell component. Am J Surg Pathol 2015;39:338-348. 